\title{
Utilizando a Norma ISO/IEC 14598-5 na Avaliação de Qualidade de Hiperdocumentos Web
}

\author{
Renata Pontin de M. Fortes ${ }^{1}$ Elisandra Aparecida da Silva Débora Maria Barroso Paiva ${ }^{1}$ \\ renata@icmc.usp.br \\ elisandra@tecban.com.br \\ debora@icmc.usp.br
}

\author{
Departamento de Ciências de Computação e Estatística \\ Instituto de Ciências Matemáticas e de Computação - Universidade de São Paulo \\ Caixa Postal 668 - São Carlos (SP)
}

\begin{abstract}
Resumo
Este artigo descreve como a Norma ISO/IEC 14598-5 pode ser utilizada na avaliação de qualidade de hiperdocumentos web. Devido a sua generalidade, observa-se a necessidade de considerar aspectos inerentes ao tipo da aplicação. Foi realizado um estudo de caso em que hiperdocumentos web foram avaliados considerandose métricas estruturais. A abordagem utilizada no estudo de caso inclui Sistemas de Aprendizado de Máquina de forma a ajudar webmasters a interpretar os resultados obtidos.

Palavras-chave: hiperdocumentos web, métricas estruturais
\end{abstract}

\begin{abstract}
This paper describes as ISO/IEC 14598-5 Standard can be utilized on web hyperdocuments quality evaluation. Aspects of application should be respected because the Standard is generic. It was made a case study in that web hyperdocuments have been evaluated regarding to structural metrics. The evaluating approach includes Machine Learning Systems to provide webmasters a better interpretation regarding to gathered results.
\end{abstract}

Keywords: web hyperdocuments, structural metrics

\section{Introdução}

O avanço da $W e b$ tornou a atividade de construção de hiperdocumentos amplamente praticada. Esta prática proporcionou uma rápida e bastante difundida utilização dos recursos na Web para disponibilização de Sistemas de Informação, denominados WIS (Web Information Systems), para diversos domínios de aplicação. Tais aplicações, cunhadas WebApps (Web Applications) por Pressman [26], requerem também processos de desenvolvimento, e para tanto, a Engenharia na $W e b$ se apresenta com diversas influências da própria Engenharia de Software.

Um produto de qualidade é, geralmente, o objetivo principal de um processo "produtivo" de desenvolvimento. Neste artigo será focalizado o tema qualidade sob a perspectiva de software, de WebApps e de hiperdocumentos web. Foi verificado que, para se alcançar o objetivo de se obter um produto com qualidade, a aplicação de métricas é uma das atividades que podem ser consideradas. Segundo Mendes et al. [15], a utilização de métricas é uma abordagem apropriada para avaliar a qualidade e o desenvolvimento de WebApps, assim como para obter feedback de maneira a auxiliar o entendimento, controle, melhorias, e prognósticos sobre tais produtos e processos. No entanto, somente aplicar métricas e coletar resultados não é suficiente para o processo de avaliação de qualidade de um produto de software. É necessário utilizar uma metodologia sistemática na qual a aplicação de tais métricas seja umas das atividades a serem cumpridas. $\mathrm{O}$ objetivo é garantir que a avaliação de

\footnotetext{
${ }^{1}$ Suporte parcial do CNPq - Projeto InCA-SERVE - Processo nro.680069/00-3.
} 
qualidade seja planejada de forma a fornecer resultados confiáveis. Dessa forma, esse artigo tem por objetivo apresentar como a Norma ISO/IEC 14598-5 [12] pode ser utilizada na avaliação de qualidade de hiperdocumentos web, descrevendo como as etapas propostas pela norma podem ser executadas para sua avaliação. A documentação obtida a partir da aplicação da norma permite que todos os interessados nos resultados possam conhecer sob quais condições e em qual contexto a avaliação é realizada. Um estudo de caso em que foi avaliada a qualidade da estrutura (propriedades de links) de hiperdocumentos web foi realizado de forma a subsidiar a utilização da abordagem descrita. Os resultados obtidos das métricas indicaram quais possíveis características de qualidade dos hiperdocumentos web são representativas. Deve-se ressaltar que para a coleta de métricas relacionadas à estrutura de hiperdocumentos web, para efeito de obtenção de um número mais estatisticamente representativo, se restringiu aos seus links estáticos.

A Seção 2 faz uma breve revisão sobre avaliação de qualidade de software; a Seção 3 apresenta como a norma ISO/IEC 14598-5 pode ser utilizada na avaliação de hiperdocumentos web; a Seção 4 se refere ao estudo de caso realizado para avaliar a qualidade de hiperdocumentos web em relação à estruturação e a Seção 5 apresenta as conclusões.

\section{Avaliação de Qualidade de Software}

Diversos métodos e processos para avaliação de qualidade de software têm sido propostos ultimamente como conseqüência da necessidade de se garantir que os produtos de software satisfaçam os requisitos de qualidade relevantes para o domínio ao qual pertencem. Como exemplos, podem ser citados: (1) o método Squid - Software Quality in Development [2], pelo qual uma organização desenvolvedora de software pode usar medições para planejar e controlar a qualidade do produto durante o desenvolvimento, avaliar a qualidade do produto final e aprender sobre o processo de software e sobre o produto e (2) o conjunto de normas ISO/IEC 14589, que estabelece como deve ser realizado o processo de avaliação de produtos de software considerando diferentes perspectivas. Em uma aplicação anterior do conjunto de normas ISO/IEC 14589 para avaliação de um sistema hipermídia educacional [25] foi observada a sua potencialidade como auxílio prático no planejamento das etapas de avaliação.

De forma geral, qualidade de software pode ser vista como um conjunto de características que devem ser alcançadas em um determinado grau para que o produto atenda às necessidades de seus usuários. É por meio desse conjunto de características que a qualidade do software pode ser descrita e avaliada.

A avaliação de produtos de software requer planejamento, controle e uso de técnicas de avaliação adequadas. Medições podem ser realizadas para planejar e controlar a qualidade do produto durante o desenvolvimento, avaliar a qualidade do produto final e aprender sobre o processo de software [27].

Em particular, a norma ISO/IEC 14598-5 [12] fornece um conjunto de recomendações que orienta o planejamento e a execução de um processo de avaliação de um software, definindo as atividades que devem ser executadas. O processo de avaliação proposto pode ser utilizado (1) para avaliar produtos já existentes ou em desenvolvimento, independentemente do domínio ao qual pertençam ou (2) por avaliadores em laboratório, fornecedores ou compradores de software, usuários e entidades certificadoras, cada qual com seu objetivo. Estes dois fatores demonstram a generalidade da norma e, portanto, a necessidade de adaptála ao contexto do software que será avaliado. 
A norma sugere que cinco etapas sejam executadas durante o processo de avaliação. A primeira delas refere-se à Análise de Requisitos, em que são descritos os objetivos da avaliação, definindo-se os requisitos a serem avaliados. Vários pontos de vista podem ser considerados, dependendo dos diferentes usuários do produto. A etapa de Especificação da Avaliação tem por objetivo a definição do escopo da avaliação e as medições a serem executadas no produto submetido à avaliação nos seus vários componentes. São definidas também as restrições, os métodos a serem utilizados e as responsabilidades de todos os envolvidos no processo de avaliação. Na etapa de Planejamento da Avaliação são documentados os procedimentos a serem usados pelo avaliador para executar as medições especificadas na fase anterior. $\mathrm{O}$ avaliador deve produzir um plano que descreva os recursos necessários para realizar a avaliação especificada, a distribuição desses recursos nas várias ações a serem executadas bem como os prazos, a equipe de avaliação, os riscos associados e todas as atividades envolvidas. Na etapa de Execução da Avaliação são obtidos resultados da execução de ações para medir e verificar o produto de software de acordo com os requisitos, com a especificação e com o projeto da avaliação. Ao final dessa etapa obtém-se o rascunho do relatório e dos registros da avaliação. $\mathrm{Na}$ etapa de Conclusão da Avaliação deve-se revisar o relatório da avaliação e disponibilizar os dados resultantes da mesma.

Considerando-se que a expansão da web gerou um aumento significativo no desenvolvimento de hiperdocumentos e que os mesmos devem ser avaliados em relação à implementação de atributos de qualidade (assim como qualquer outra aplicação), a seção seguinte descreve como a norma ISO/IEC $14598-5$ pode ser utilizada na avaliação de hiperdocumentos web.

\section{Utilização da norma ISO/IEC 14598-5 na avaliação de hiperdocumentos web}

O desenvolvimento de sites e de WebApps tem sido um desafio, não somente do ponto de vista de processos e tecnologias utilizadas, mas também no que se refere à garantia de qualidade. Particularmente, em relação a esta última perspectiva, é necessário que se tenha uma definição clara de requisitos funcionais e não-funcionais que devem ser implementados, de forma que seja possível medir, controlar e melhorar a qualidade do produto. Por exemplo, o método Web-QEM para avaliação de WebApps por avaliadores-especialistas, foi proposto por Olsina [19, 20, 21, 22, 23] com base na norma ISO 14598-5. Trata-se de um método quantitativo para avaliação de qualidade de sites e WebApps. Tem sido investigada a sua utilização com um suporte automatizado [24].

Algumas características específicas de hiperdocumentos web são a combinação de diferentes mídias e tecnologias para apresentar informações, bem como a estruturação interna e externa aos documentos, por meio de links. Quanto à característica de estruturação, os hiperdocumentos web apresentam semelhança aos softwares de tempos remotos (mencionados por Pressman, como tangled Web). O uso não sistemático dessas características pode causar problemas para visitantes que desejam encontrar informações específicas. Por exemplo, a falta de qualidade de um hiperdocumento pode estar associada à falta de um esquema de organização claro da página principal e, à existência de links quebrados ou inválidos, os quais podem levar os usuários a terem má impressão do site e da instituição a que se referem [18].

Conforme visto na seção anterior, a norma ISO/IEC 14598-5 apresenta as etapas que devem ser executadas em uma avaliação. Devido a sua generalidade, deve ser utilizada considerando-se as características inerentes ao domínio para o qual a avaliação será realizada e os requisitos da própria aplicação. Dessa forma, é apresentado a seguir como esta norma 
pode ser utilizada na avaliação de hiperdocumentos web, de forma a demonstrar uma possível alternativa para a execução das etapas sugeridas e os principais parâmetros que devem ser considerados. Como a atividade de produção de hiperdocumentos web geralmente envolve a manipulação de um volume grande de informações (entre páginas e links), se não for abordada sistemática e cuidadosamente, problemas de qualidade indesejáveis podem acarretar que suas informações sejam interpretadas erroneamente, com conseqüências de que organismos governamentais criem regulamentação e legislação com relação à liberdade de autoria na web, o que levaria a uma irreparável perda de expressão na Internet.

\subsection{Análise de Requisitos}

Por se tratar de elemento de software, normalmente esta etapa é realizada considerando-se o modelo de qualidade descrito na norma ISO/IEC 9126-1 [13]. No entanto, quando um requisito se refere a características não definidas por essa norma, deve ser feita referência à literatura que o define [12]. Os requisitos de qualidade especificados na etapa de análise de requisitos durante o desenvolvimento do sistema devem ser considerados na avaliação. $\mathrm{O}$ avaliador deve considerar que, ao se desenvolver um produto de software (neste caso, hiperdocumentos web), o objetivo não é alcançar a qualidade perfeita, mas sim a qualidade necessária e suficiente para o uso especificado, quando o produto final for disponibilizado e realmente utilizado [6]. Portanto, é importante que ele identifique quais são os requisitos de qualidade necessários para o(s) hiperdocumento(s) que será(ão) avaliado(s) e decidir em que grau essas características precisam ser alcançadas para satisfazer as necessidades dos usuários. Por exemplo, hiperdocumentos de um site de comércio eletrônico devem possuir, sobretudo, confiabilidade (no sentido de oferecer segurança nas transações efetuadas) mas também, facilidade de uso, navegabilidade, eficiência, dentre outros [1]. Por outro lado, sites educacionais devem privilegiar a estruturação dos hiperdocumentos e a atualização e abrangência das informações disponibilizadas [4].

\subsection{Especificação da Avaliação}

Nesta etapa são definidos o escopo e as medições que devem ser executadas nos hiperdocumentos web. Em relação ao escopo da avaliação, devem ser identificados os componentes que serão avaliados. Definir o escopo é particularmente importante quando o produto submetido à avaliação é parte de um sistema constituído por software, hardware, redes e outras organizações, pois a separação entre tais entidades nem sempre é óbvia [12].

$\mathrm{O}$ avaliador deve especificar as medições que serão executadas nos componentes selecionados para avaliar a implementação dos requisitos estabelecidos na etapa de análise de requisitos. Estas especificações devem ser formuladas, basicamente, como uma combinação dos seguintes tipos de estruturas: (1) especificação formalizada de uma métrica que deverá ser aplicada no produto (ou nos componentes) avaliado, juntamente com instruções para apresentação dos resultados obtidos no relatório de avaliação e (2) uma referência a estruturas nos componentes que serão avaliados de forma a especificar os requisitos que serão verificados nessas estruturas.

No caso de hiperdocumentos web, podem ser avaliadas, por exemplo, as características e condições dos links (este seria um requisito de avaliação), permitindo identificar o número de links quebrados, a quantidade de links e a quantidade de imagens que são links, de forma que os dados obtidos possam ser analisados durante as etapas de autoria e manutenção. Neste 
caso, métricas propostas na literatura que promovam a avaliação de qualidade da utilização de links no hiperdocumento web, como aquelas propostas por Olsina et al [18], devem ser utilizadas e especificadas nesta etapa.

\subsection{Planejamento da Avaliação}

O planejamento da avaliação deve documentar os procedimentos a serem utilizados pelo avaliador para executar as medições especificadas na etapa de especificação da avaliação. $\mathrm{O}$ avaliador deve produzir um plano que descreva os recursos necessários para executar a avaliação especificada. Esta etapa envolve a execução de três sub-atividades [12]:

- Documentação dos métodos de avaliação utilizados: o avaliador deve documentar detalhadamente os métodos a serem aplicados para executar as medições especificadas nos componentes que serão avaliados;

- Otimização do plano de avaliação: o avaliador deve relacionar os métodos de avaliação aos elementos da especificação da avaliação que, por sua vez, estão relacionados aos requisitos definidos na etapa de análise de requisitos;

- Definição das atividades de avaliação com relação aos recursos disponíveis: o avaliador deve planejar a execução de atividades considerando recursos humanos e recursos de software e hardware.

Considerando-se novamente o exemplo da seção anterior, em que foi sugerida a utilização de métricas para avaliação das condições dos links de hiperdocumentos web, na etapa de planejamento da avaliação deveriam ser definidos quais os métodos seriam utilizados para possibilitar a aplicação das métricas e como estes métodos seriam aplicados. Uma possibilidade seria a utilização de métricas automatizadas que pudessem auxiliar a coleta de dados. Neste caso, a utilização dessas métricas deveria ser planejada, definindo-se os componentes para os quais poderiam ser aplicadas. Além disso, deveria ser definido quem estaria responsável pela aplicação das métricas, em quantas seções a coleta de dados seria realizada, e quais seriam os requisitos de software e hardware necessários para a realização da avaliação.

\subsection{Execução e Conclusão da Avaliação}

$\mathrm{Na}$ etapa de execução da avaliação, são cumpridas todas as atividades planejadas considerando-se as etapas realizadas anteriormente. A etapa de conclusão da avaliação consiste na elaboração de relatórios que registrem os dados obtidos a partir da execução da avaliação.

Utilizando-se o processo proposto pela norma discutida nesta seção, foi realizado um estudo de caso no qual foi avaliada a estrutura de hiperdocumentos web, ou seja, foram avaliadas as propriedades de links de forma que eles pudessem ser classificados e, assim, informações sobre a estrutura do hiperdocumento pudessem ser obtidas para serem consideradas nas futuras manutenções.

\section{Estudo de caso: Avaliação da estrutura de hiperdocumentos utilizando a Norma ISO/IEC 14598-5}

É apresentado a seguir como a Norma ISO/IEC 14598-5 foi utilizada de forma a possibilitar a realização da avaliação da estrutura de hiperdocumentos web. 


\subsection{Análise de Requisitos e Especificação da Avaliação}

$\mathrm{Na}$ etapa de análise de requisitos foi definido o objetivo geral da avaliação: analisar a qualidade da estrutura de hiperdocumentos web. Como requisitos de avaliação, foram definidos os fatores de qualidade manutenibilidade e legibilidade dos hiperdocumentos. $\mathrm{O}$ fator manutenibilidade é definido como o conjunto de atributos que indicam o esforço necessário para fazer modificações especificadas no software devido a mudanças de ambiente, ou nos seus requisitos [13]. O fator de qualidade legibilidade é um conceito que deve indicar a facilidade de se "ler" um hiperdocumento. Esse fator de qualidade representa o esforço requerido do usuário para "ler" o hiperdocumento, ou seja, para navegar sobre ele e entender a estrutura das idéias nele contidas. $\mathrm{O}$ atributo legibilidade foi considerado como fator de qualidade por Hatzimanikatis et al [10].

$\mathrm{Na}$ etapa de especificação da avaliação foi definido primeiramente o escopo da avaliação, tendo sido estabelecido que seriam avaliadas somente as propriedades dos links.

Em seguida, foram definidas e especificadas as métricas que seriam utilizadas para avaliar a implementação dos requisitos de manutenibilidade e legibilidade. As métricas selecionadas foram divididas em Métricas aplicadas a sites e Métricas aplicadas a páginas. $\mathrm{Na}$ primeira categoria, as métricas foram computadas, quando a estrutura de todo $\mathrm{o}$ hiperdocumento web já era conhecida. As métricas da segunda categoria foram computadas a partir de conhecidos os dados das páginas da web.

As métricas aplicadas a páginas necessitavam dos dados provenientes das páginas dos sites (nós do grafo). Essas métricas foram: Número de links que eram imagens, Superfície dessas imagens, Número de In Links e Número de Out Links. Essas métricas necessitavam das informações presentes apenas nos links das páginas, tais como: âncora e página-destino. As métricas pertencentes a esta categoria foram especificadas, como apresentado a seguir:

\section{Número de links que são imagens}

Essa métrica define o número de links que são imagens em uma página. Conforme tem sido observado, com os recursos de multimídia disponíveis, a "iconização" para representar direções dos links ou "localização" de mais informação a ser explorada pelo usuário tem se popularizado. Isso é potencialmente bom, pois facilita a leitura para o usuário, mas quando não possui uniformidade, acaba levando à inconsistência e pode desorientar o usuário.

\section{Superficie dessas imagens}

Essa métrica define a superfície total utilizada pelos links que contêm imagens em uma página. A métrica não reflete se as imagens são "pesadas" ou não em termos de espaço em memória, ela apenas mostra se as imagens ocupam uma quantidade razoável ou não da superfície em tela.

\section{Número de In Links}

Essa métrica define o número de in links em uma página, ou seja, aqueles links que apontam para uma página do mesmo site do hiperdocumento. In links podem ser muito convenientes porque eles permitem manter o texto "amigável" e local, apresentando a informação de uma maneira acessível e não restringindo a informação em apenas uma página. 


\section{Número de Out Links}

Essa métrica define o número de out links em uma página, isto é, aqueles links que apontam para páginas fora do site do hiperdocumento. Se o número de out links é alto, então mais cuidado é requerido do mantenedor, pois não há controle "eficiente" no caso desses links apontarem para páginas que podem ser removidas aleatoriamente.

As métricas aplicadas a sites foram as que puderam ser aplicadas a websites, ou seja, computado seu valor a partir de conhecida a estrutura de todo o hiperdocumento. As métricas pertencentes a esta categoria foram especificadas, e são resumidamente apresentadas a seguir. A especificação completa dessas métricas pode ser encontrada em [3] e [7].

\section{Compactação}

Indica a conectividade intrínseca do hiperdocumento. Para o leitor, um alto valor de compactação indica que cada nó possui muitos links.

\section{Estratificação}

É uma métrica usada para capturar a ordenação linear do hiperdocumento. Essa métrica reflete as escolhas de navegação do usuário enquanto navega no hiperdocumento. Dependendo do modo como o hiperdocumento é construído, os usuários terão acesso a uma estrutura mais ou menos flexível de navegação. Um hiperdocumento estratificado não permite muita flexibilidade durante a navegação, ou seja, fornece um modo estratificado ou hierárquico de navegação. Um hiperdocumento com uma alta estratificação indica que os usuários não têm muita escolha e, conseqüentemente, têm menor possibilidade de sofrer com o problema de desorientação. Por outro lado, uma baixa estratificação sugere que o número excessivo de links que o usuário possa escolher pode causar desorientação ao usuário [9].

\section{Impureza da árvore}

É a proporção de desvio do grafo do hiperdocumento quando comparado com uma estrutura do tipo árvore pura (que não possui ciclos). A estrutura de documentos tradicionais impressos é muito semelhante a de uma estrutura de árvore pura e, geralmente, pode ser facilmente compreendida tanto pelos leitores como pelos mantenedores.

\subsection{Planejamento da Avaliação}

Devido ao fato de as métricas especificadas serem de difícil interpretação, foi planejada a execução dos experimentos com o suporte de Sistemas de Aprendizado de Máquina (AM). Com este suporte, os experimentos teriam a análise de dados obtidos facilitada. Foi definida também a utilização da ferramenta DB-LiOS ${ }^{2}$ (Database Link Oriented System) [29], de forma a possibilitar a extração de informações necessárias à coleta de valores para as métricas.

A etapa de planejamento definiu a metodologia utilizada na avaliação, composta pelas tapas apresentadas a seguir.

Primeiramente deveria ser obtida, através da utilização da ferramenta DB-LiOS, uma base de dados processada, constituída por exemplos no formato atributo-valor. Em seguida

\footnotetext{
${ }^{2}$ A ferramenta DB-LiOS foi desenvolvida com o objetivo de proporcionar uma avaliação automática da consistência estrutural de websites através de extração e classificação de seus links, segundo as métricas baseadas em reuso de links [8].
} 
essa base de dados deveria ser submetida a um processamento realizado pelo algoritmo de AM não supervisionado denominado Autoclass [5], que é responsável por descobrir clusters presentes na base de dados. Com os resultados obtidos (clusters encontrados), deveria ser realizado um processamento, por um mecanismo computável, com o objetivo de rotular os exemplos da base original com o cluster ao qual pertencem, gerando uma base de dados com uma dimensão adicional. Para isso, foi planejada a utilização da ferramenta InClass [14]. Essa ferramenta utiliza como entrada um dos relatórios gerados por Autoclass e o conjunto de exemplos originais (não rotulados). A saída gerada por InClass é o conjunto de exemplos contendo agora uma dimensão adicional, pois contém o atributo classe relacionado ao cluster ao qual pertence cada exemplo. Neste ponto, a nova base de dados possui as características necessárias para ser utilizada como entrada para algoritmos de AM supervisionados.

Como o principal interesse foi tentar explicar clusters previamente encontrados, a linguagem de descrição de conceitos (ou hipóteses) utilizada pelo algoritmo de AM supervisionado escolhido deveria ser uma linguagem simbólica, tal como regras ou árvores de decisão. Então, foi escolhido o See5 como algoritmo de AM supervisionado. Assim, a base de dados gerada por InClass, no formato requerido por See5, poderia ser processada para induzir regras de conhecimento. Tomando como base as regras geradas pelo See5, análise de erro e diversas estatísticas, o especialista é capaz de realizar uma análise apurada para tentar explicar o agrupamento dos exemplos nos clusters encontrados. $\mathrm{O}$ processo seria então finalizado com a análise do especialista sobre as regras de conhecimento geradas. Através dessa análise, que realmente seria possível se verificar se o conhecimento gerado é relevante, desconhecido ou útil.

Além disso, foi definido na etapa de planejamento que os experimentos seriam realizados com no mínimo 20 sites de instituições de ensino superior. Esses sites teriam que possuir um número mínimo de páginas (40), pois os algoritmos de AM requerem um número razoável de exemplos para obter bons resultados. Foi planejada a realização dos experimentos em um laboratório de Computação, por uma aluna de mestrado, utilizando um único computador (Pentium II, $400 \mathrm{MHZ}, 128 \mathrm{MB}$ memória RAM) e utilizando a plataforma Windows.

\subsection{Execução da Avaliação}

Nesta etapa, foram realizados os procedimentos definidos na etapa de planejamento da avaliação, considerando-se o que foi estabelecido nas etapas de especificação da avaliação e análise de requisitos [30].

Inicialmente, a ferramenta DB-LiOS foi utilizada para extrair as informações necessárias para aplicação dos módulos responsáveis pela coleta das métricas de sites e páginas. Esta etapa considerou as especificidades características de estruturação por meio de links web. Assim, observou-se que os links disponibilizados na web são os elementos de interação do usuário com o sistema de navegação (browser), que não são definidos na interface do sistema, mas sim no conteúdo das páginas do hiperdocumento. Os links, de forma geral, definidos e embutidos nas páginas de um website, definem a estrutura de ligação entre as páginas por meio dos seguintes componentes: 1) página onde se encontra o link ("página origem"); 2) rótulo que identifica a presença do link na página origem ("âncora"), que pode se constituir de um texto ou mesmo uma imagem; e 3) página para onde o link aponta ("página destino") que pode ser simplesmente um direcionamento para um outro ponto na própria página ou uma outra página. Os dados coletados pela ferramenta DB-LiOS são extraídos por 
um crawler que foi implementado para extrair os componentes de links das páginas de websites.

O crawler implementado na ferramenta DB-LiOS percorre somente as páginas que estão abaixo da hierarquia de um determinado diretório, pertencente ao servidor de arquivos de um website. Assim, o crawler não extrai os links de subdomínios de um site, por exemplo, para o site "http://www.icmc.sc.usp.br/" existem alguns subdomínios como: "http://nt-labes. icmc.sc.usp.br/", "http://labic.icmc.sc.usp.br/", "http://java.icmc.sc.usp.br/", "http://gbdi.icmc.sc.usp.br/", e seus links não são extraídos pela ferramenta DB-LiOS quando iniciamos a extração a partir do site "http://www.icmc.sc.usp.br". Embora tal consideração seja uma garantia de "escopo" de varredura de um website, potencialmente ela ocasiona uma restrição em sites que adotam particionamento intensivo das informações em subdomínios. Os frames são coletados pelo crawler como páginas. Frames são divisões da tela do browser em diversos "quadros". Com isso, é possível apresentar mais de uma página simultaneamente numa janela do browser. Os frames apresentam vários problemas e coletar a composição exata com que os frames se dispõem no browser em tempo-de-execução é uma tarefa difícil.

Apesar de ser fácil colocar frames em páginas, nem todos os usuários gostam do uso de tal recurso [11]. De acordo com Nielsen [16], organizar uma página em frames pode confundir o usuário uma vez que o uso de frames quebra o modelo fundamental do usuário de página web. Mais recentemente, [17] reviu alguns problemas relacionados a frames e ponderou que frames não são mais o "desastre" que pareciam na época em que surgiram. Devido a alguns avanços na tecnologia de browsers, foram reduzidos os problemas de navegação, impressão e de utilização de marcadores de páginas. Mas segundo Nielsen existem ainda alguns problemas com frames: as URLs não funcionam "transparentemente" e o uso de frames torna a interação com a página mais difícil. A não "transparência" significa que uma vez que a informação de endereçamento apresentada no topo da tela do browser, ela não constitui uma especificação completa da informação apresentada na tela. Se um autor copia uma URL de forma a incluí-la como um link em uma de suas páginas, então aquele link não levará o usuário para a visão desejada, mas para o estado inicial do conjunto de frames.

A Tabela 1 apresenta a razão do número de páginas em cada um dos hiperdocumentos dos sites avaliados no experimento, pelo número de links estáticos encontrados. Como a ferramenta DB-LiOS captura somente links estáticos, não foram contabilizados os links dinâmicos, ou seja, aqueles que não possuíam tags $<$ a href $>$ em sua definição. Embora esta restrição da ferramenta possa refletir um número baixo de links nos hiperdocumentos web da atualidade, como pode ser visto nas razões apresentadas na terceira coluna da Tabela 1, o objetivo do experimento foi o de verificar as métricas clássicas de estruturação de hipertextos no ambiente web.

Foram coletados valores para as métricas In Links, Out Links, Número de links que são imagens e Superfície dessas imagens. Seus valores puderam ser obtidos verificando apenas a página-destino e âncora do link. Com a página-destino é possível saber se o link é um link que aponta para uma página de outro site, e com a âncora é possível saber se é uma imagem. Essas métricas aplicadas a páginas são calculadas por meio de contadores que somam as ocorrências de suas características.

A ferramenta DB-LiOS, utilizada para extrair os links dos hiperdocumentos web fornecidos pelo usuário, procura por links da seguinte forma:

" $<$ a href= "http://www..." $>$ Pequeno texto $</ a>$ 
Para obter o número de links que são imagens são percorridas todas as âncoras fornecidas pela ferramenta DB-LiOS, procurando por '<img' na primeira posição da string representando a âncora, da seguinte forma:

$<$ a href="http://www..." $><$ img src="... .gif" width="125" height="78" border="0" $></ a>$

\section{Tabela 1 - Sites utilizados no experimento}

\begin{tabular}{|l|l|r|}
\hline nro & Hiperdocumento Web & $\begin{array}{c}\text { nro de páginas/ } \\
\text { nro de links }\end{array}$ \\
\hline \hline 1 & www.ufal.br & 0,235 \\
\hline 2 & www.uesb.br & 0,316 \\
\hline 3 & www.ufba.br & 0,152 \\
\hline 4 & www.uece.br & 0,316 \\
\hline 5 & www.ufes.br & 0,131 \\
\hline 6 & www.pucsp.br & 0,244 \\
\hline 7 & www.unimontes.br & 0,437 \\
\hline 8 & www.unisul.rct-sc.br & 0,088 \\
\hline 9 & www.ime.eb.br & 0,349 \\
\hline 10 & www.eep.br & 0,816 \\
\hline 11 & www.fafica.br & 0,281 \\
\hline 12 & www.ufv.br & 0,175 \\
\hline 13 & www.ufpb.br & 0,130 \\
\hline 14 & www.ufpe.br & 0,177 \\
\hline 15 & www.ita.cta.br & 0,033 \\
\hline 16 & www.ufop.br & 0,110 \\
\hline 17 & www.ufpa.br & 0,189 \\
\hline 18 & www.puc-rio.br & 0,090 \\
\hline 19 & www.puc-campinas.br & 0,297 \\
\hline 20 & www.unaerp.br & 0,043 \\
\hline 21 & www.ufma.br & 0,149 \\
\hline 22 & www.ufmt.br & 0,353 \\
\hline 23 & www.ufmg.br & 0,103 \\
\hline 24 & www.ufr.br & 0,094 \\
\hline 25 & www.icmc.usp.br & 0,070 \\
\hline
\end{tabular}

Quando a âncora se trata de uma imagem, são considerados os valores de width $\mathrm{e}$ height e o seguinte cálculo é realizado para obter a superfície utilizada por essas imagens:

$$
\sum_{i=1}^{L} \text { width } \times \text { height }
$$

em que L é o número de links em de uma página.

Para verificar o número de In Links em cada página, é considerada a string que representa a página-destino e verifica-se se a string contém o site onde a página-origem está localizada. Para verificar o número de Out Links em cada página, é considerada a string que representa a página-destino e verifica-se se a string não contém o site onde a página-origem está localizada. Portanto aponta para uma página de outro site.

Em relação às métricas aplicadas a sites, primeiramente foram coletados dados para Compactação, Estratificação e Impureza da árvore, como definido na etapa de planejamento da avaliação (Seção 4.2). Para isso, foi utilizada a ferramenta DB-LiOS, que forneceu resultados no formato atributo-valor. Os resultados obtidos (base de dados) foram submetidos 
ao AutoClass, que descobriu 2 clusters na base de dados. Utilizando o Inclass para o conjunto de dados obtidos a partir da aplicação do AutoClass, foram obtidos os resultados apresentados na Tabela 2, no formato requerido pelo See5.

Tabela 2 - Resultados obtidos a partir da utilização do Inclass

\begin{tabular}{|c|c|c|c|c|}
\hline $\begin{array}{c}\text { Hiperdocumento } \\
\text { Web }\end{array}$ & Compactação & Estratificação & Impureza da árvore & Classe \\
\hline 1 & 0.22 & 0.32 & 0.04 & Classe_0 \\
\hline 2 & 0.01 & 0.07 & 0.02 & Classe_0 \\
\hline 3 & 0.09 & 0.29 & 0.01 & Classe_1 \\
\hline 4 & 0.01 & 0.09 & 0.02 & Classe_0 \\
\hline 5 & 0.21 & 0.94 & 0.02 & Classe_0 \\
\hline 6 & 0.07 & 0.39 & 0.17 & Classe_0 \\
\hline 7 & 0.28 & 0.23 & 0.02 & Classe_0 \\
\hline 8 & 0.93 & 0.32 & 0.23 & Classe_0 \\
\hline 9 & 0.03 & 0.21 & 0.03 & Classe_0 \\
\hline 10 & 0.03 & 0.19 & 0.01 & Classe_1 \\
\hline 11 & 0.26 & 0.41 & 0.11 & Classe_0 \\
\hline 12 & 0.11 & 0.15 & 0.01 & Classe_1 \\
\hline 13 & 0.06 & 0.30 & 0.25 & Classe_0 \\
\hline 14 & 0.16 & 0.59 & 0.08 & Classe_0 \\
\hline 15 & 0.46 & 1.39 & 0.58 & Classe_0 \\
\hline 16 & 0.51 & 1.17 & 0.05 & Classe_0 \\
\hline 17 & 0.14 & 0.59 & 0.01 & Classe_1 \\
\hline 18 & 0.55 & 0.58 & 0.06 & Classe_0 \\
\hline 19 & 0.04 & 0.13 & 0.01 & Classe_1 \\
\hline 20 & 0.79 & 0.23 & 0.30 & Classe_0 \\
\hline 21 & 0.29 & 0.94 & 0.07 & Classe_0 \\
\hline 22 & 0.06 & 0.25 & 0.05 & Classe_0 \\
\hline 23 & 0.06 & 0.20 & 0.05 & Classe_0 \\
\hline 24 & 0.86 & 0.66 & 0.10 & Classe_0 \\
\hline 25 & 0.23 & 1.10 & 0.15 & Classe_0 \\
\hline
\end{tabular}

Esse conjunto de exemplos é utilizado por See5 para induzir um conjunto de regras não ordenadas if-then. O conjunto de regras induzidas por See5 foi então analisado para tentar encontrar um significado semântico para o nome dos clusters. O processo efetuado para auxiliar a análise de clusters identificados automaticamente é ilustrado na Figura $1 . \mathrm{Na}$ próxima seção são descritos os resultados obtidos.

\subsection{Conclusão da Avaliação}

Nesta seção são apresentados os resultados do experimento. Esse experimento foi realizado em duas etapas, uma com os dados das páginas e outra com os dados de sites.

\subsubsection{Experimento com os dados das páginas}

O experimento realizado com os dados coletados das páginas não ofereceu resultados tão significativos quanto aqueles oferecidos pela coleta de dados dos sites. O atributo presente na maioria das regras e com maior importância foi o atributo Superfície das imagens, que 
indica a superfície utilizada pelas imagens em uma dada página. Pretende-se realizar futuramente outras experiências com esses dados, utilizando indução construtiva que permite criar novos atributos como uma combinação dos atributos existentes [28].

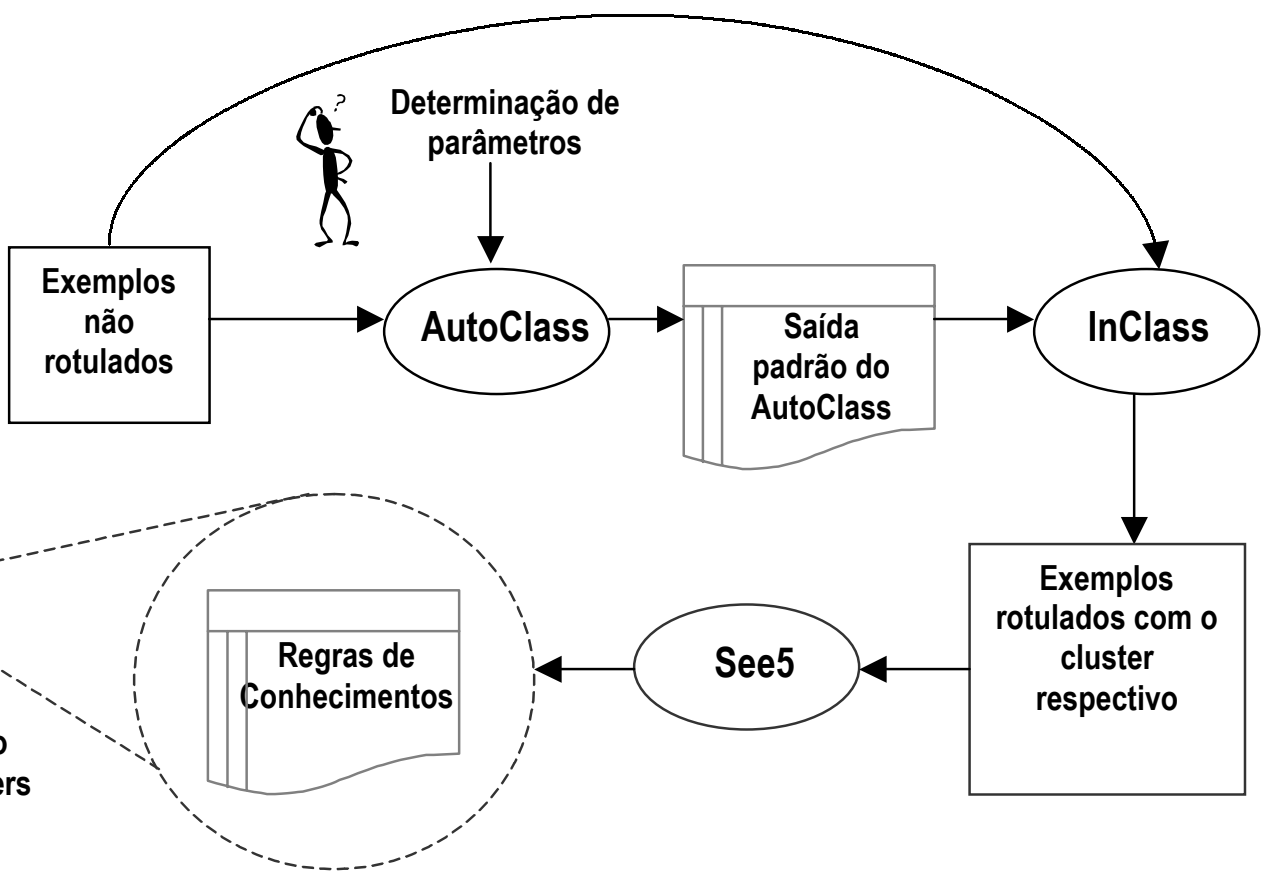

Figura 1 - Processo utilizado para explicação dos clusters

\subsubsection{Experimento com os dados de sites}

A base de dados de todos os sites foi submetida ao Autoclass sem fixar um número de clusters, tendo sido encontrados 2 clusters. A Tabela 3 apresenta um resumo dos resultados obtidos, em que:

- Clusters - representa os clusters;

- \# Exemplos - número de exemplos na base de dados;

- \% Classe - porcentagem de exemplos pertencentes ao cluster;

- Erro aparente - erro aparente de See5, isto é, utilizando toda a base de dados de treinamento e teste;

- Erro verdadeiro (10CV) - erro verdadeiro obtido através de 10k-fold cross-validation;

- Erro CM - erro da classe majoritária;

- \# Regras - número de regras encontradas por See5;

- \# Médio Regras - número médio de regras obtido nas 10 execuções de cross-validation.

Tabela 3 - Resumo dos resultados do experimento com todos os sites

\begin{tabular}{|cccccccc|}
\hline $\begin{array}{c}\# \\
\text { Exemplos }\end{array}$ & Clusters & $\begin{array}{c}\% \\
\text { Classe }\end{array}$ & $\begin{array}{c}\text { Erro } \\
\text { Aparente }\end{array}$ & $\begin{array}{c}\text { Erro (10 CV) } \\
\text { Verdadeiro }\end{array}$ & Erro CM & $\begin{array}{c}\text { \# } \\
\text { Regras }\end{array}$ & $\begin{array}{c}\text { \# Médio } \\
\text { Regras }\end{array}$ \\
\hline \hline 25 & $\mathrm{C}(0)$ & $80 \%$ & $0,0 \%$ & $0,0 \% \pm 0,0 \%$ & $20 \%$ & 2 & $2 \pm 0$ \\
\hline
\end{tabular}


As regras obtidas são apresentadas na Figura 2. É importante notar que essas regras classificam os exemplos de acordo com o atributo Impureza da árvore. Impureza da árvore é um critério que influencia a legibilidade de hiperdocumentos. Navegação em um hiperdocumento web com estrutura de árvore é mais fácil que navegação em hiperdocumentos com muitas referências cruzadas. A segunda regra classifica cinco sites com um valor menor ou igual a 0.01 (20\% dos exemplos). Os outros sites são classificados pela primeira regra (Impureza da árvore maior que 0.01). Nessas regras aparece apenas o atributo Impureza da árvore, o que indica que somente esse atributo consegue diferenciar os exemplos (sites) considerados.

Rule 1: (cover 20)

Impureza da árvore $>0.01$

$\rightarrow$ classe Classe_0 [0.955]
Rule 2: (cover 5)

Impureza da árvore $<=0.01$

$\rightarrow$ classe Classe_1 [0.857]

\section{Figura 2 - Regras extraídas para todos os sites}

\section{Conclusões}

Este artigo apresentou uma abordagem para avaliação de hiperdocumentos web baseada na utilização da Norma ISO/IEC 14598-5. Devido à generalidade dessa norma, foi destacada a necessidade de se considerar características inerentes ao domínio do sistema para o qual a avaliação foi realizada. Em particular, neste trabalho foram avaliados os atributos relacionados a links de hiperdocumentos web, considerando-se os fatores de qualidade manutenibilidade e legibilidade. Em especial, procurou-se verificar as métricas clássicas de estruturação de hipertextos no ambiente web.

$\mathrm{Na}$ etapa de especificação e planejamento da avaliação foram estudadas métricas propostas na literatura para hiperdocumentos e selecionadas algumas para implementação, e coleta a partir de sites da web. Como os valores das métricas são de difícil interpretação por parte dos webmasters e não se encontram referências consolidadas a respeito de seus possíveis valores, foi apresentada uma abordagem para auxiliar a análise dos resultados. Tal abordagem contou com auxílio de técnicas de Aprendizado de Máquina, uma subárea da Inteligência Artificial que tem sido muito explorada no contexto web. A execução da avaliação, conforme planejada, foi cumprida seguindo as etapas, que tiveram seu desenvolvimento acompanhado e ainda assim as especificidades inerentes das métricas de domínio de hiperdocumentos web foram consideradas, sem prejuízo do progresso da avaliação [31].

Ao final, a principal restrição observada no estudo de caso realizado refere-se ao fato de os dados coletados para as páginas não terem oferecido resultados muito significativos. Trabalhos futuros referem-se a implementação e utilização de outras métricas no processo de avaliação de qualidade.

\section{Referências Bibliográficas}

[1] Albuquerque, A.B; Belchior, A.D. Qualidade de Websites de Comércio Eletrônico. XV Simpósio Brasileiro de Engenharia de Software - VIII Workshop de Qualidade de Software. Rio de Janeiro. Anais. p.87-99, 2001. 
[2] Boegh, J. et al. A method for software quality planning, control and evaluation. IEEE Software. Março/abril, 1999.

[3] Botafogo, R. A., Sheneiderman, B. Structural Analysis of Hypertexts: Identifying Hierarchies and Useful Metrics ACM Transactions on Information Systems, v.10, n.2, p.142-80, 1992.

[4] Campos, F.C.A. Educação a Distância e Qualidade: da Teoria à Prática. XV Simpósio Brasileiro de Engenharia de Software - VIII Workshop de Qualidade de Software. Rio de Janeiro. Anais. p.176-182, 2001.

[5] Cheesman, P., Stutz, J. Bayesian classification (autoclass): Theory and results advances in knowledge discovery and data mining. http://ic.arc.nasa.gov/ic/projects/bayes-group/autoclass-c-program.html.

[6] Collins, W.R. et al., How good is good enough. Communications of the ACM, v.37, n.1, Janeiro 1994.

[7] De Bra, P. M. E. Hypermedia Structure and Systems. Eidhoven University of Technology, 1999 (Disponível em http://wwwis.win.tue.n1/2L670/static/)

[8] Fortes, R. P. M., Nicoletti, M. C. A Family of Link Based Metrics for the Evaluation of Web Documents. SIGLINK Bulletin, v.6, n.3, p.21-23, 1997.

[9] Fortes, R. P. M.; Nicoletti, M. C. Automatic Diagnosis of Hyperdocuments Using a Family of Quantifiable Metrics. Symposium on Software Technology. Buenos Aires, Argentina. Proceedings. p.62-71, 1999.

[10] Hatzimanikatis, A. E., Tsalidis, C. T., Christodoulakis, D. Measuring the Readability and Maintainability of Hyperdocuments; J. of Software Maintenance, Research and Practice, v.7, p.77-90.

[11] Tutorial HTML do ICMC, (Disponível em http://www.icmc.sc.usp.br/manuals/HTML/)

[12] ISO/IEC 14598-5, International Standard. Information Technology - Software product evaluation - Part 5: Process for evaluators; 1997 (DIS).

[13] ISO/IEC 9126, International Standard. Information Technology - Software Product Evaluation - Quality characteristics and guidelines for their use, 1991.

[14] Martins, C. A.; Monard, M. C. Interpretação de Clusters Utilizando Aprendizado de Máquina Simbólico. 21 Iberian Latin American Congress on Computational Methods in Engineering - CILAMCE2000, Rio de Janeiro, 2000.

[15] Mendes, E., Cousell, S., Mosley, N. Measurement and Effort Prediction for Web Applications. Web Engineering 2001, p.295-310, 2001.

[16] Nielsen, J. Why frames suck most of the time, http://www.useit.com/alertbox/9612.html

[17] Nielsen, J.: Top Ten Mistakes" Revisited Three Years Later, (Disponível em http://www.useit.com/alertbox/990502.html)

[18] Olsina, L., Rodríguez, J.G., Lafuente, G., Pastor, O. Towards Automated Web Metrics. XV Simpósio Brasileiro de Engenharia de Software - VIII Workshop de Qualidade de Software. Rio de Janeiro. Anais. p.74-86, 2001

[19] Olsina, L., Web-site Quantitative Evaluation and Comparison: a Case Study o Museums, Int'l Conference on Software Engineering, ICSE '99 Workshop on Software Engineering for the Internet, Los Angeles, US, 1999 (Disponível em http://sern.cpsc.ucalgary.ca/ maurer/ICSE99WS/ICSE99WS.html ).

[20] Olsina, L., Godoy, D; Lafuente, G.J; Rossi, G. Quality Characteristics and Attributes for Academic Web Sites, WWW8 Web Engineering'99 Workshop, Toronto, Canada, 1999 (Disponível em http://budhi.uow.edu.au/webengineering99/web_engineering.html) 
[21] Olsina, L., Godoy, D; Lafuente, G.J; Rossi, G. Specifying Characteristics and Attributes for Web Sites, Proceedings of ICSE '99 Int'1 Conference on Software Engineering Web Engineering Workshop, Los Angeles, US, p.84-93, 1999.

[22] Olsina, L., Godoy, D; Lafuente, G.J; Rossi, G. Assessing the Quality of Academic Websites: a Case Study. In New Review of Hypermedia and Multimedia (NRHM) Journal Taylor Graham Publishers, UK/USA, v.5, ISSN 13614568, 1999.

[23] Olsina, L.; Rossi, G. Towards Web-site Quantitative Evaluation: defining Quality Characteristics and Attributes, Proceedings of IV Int'l WebNet Conference, World Conference on the WWW and Internet, Hawaii, USA, v.1-2, p.834-839, 1999.

[24] Olsina L., Papa, M.F., Souto, M.E., Rossi, G.; 2001, "Providing Automated Support for the Web Quality Evaluation Methodology", Proceed. of the Fourth Workshop on Web Engineering, at the 10th International WWW Conference, Hong Kong, pp. 1-11.

[25] Paiva, D.M.B.; Nunes, M.G.V.; Fortes, R.P.M. Qualidade de Produto de Software: Avaliação Prática de um Sistema de Autoria Hipermídia Educacional. VIII WQS (Workshop de Qualidade de Software) Simpósio Brasileiro de Engenharia de Software SBES'2001. Rio de Janeiro-RJ, Anais. p.114-122, 2001.

[26] Pressman, R. S. Software Engineering. $5^{\text {th }}$ edition, McGraw-Hill, 2001.

[27] Rocha, A.R.C., Maldonado, J.C, Weber, K.C. Qualidade de Software. Teoria e Prática, Prentice Hall, 2001.

[28] Russel, S. J.; Norving, P.: Artificial Intelligence: A Modern Approach. Prentice Hall, 1995.

[29] Seraphim, E.; Fortes, R. P. M DB-LiOS: Suporte Automático à Avaliação da Consistência de Links em $W W W$ XX Congresso Nacional da SBC, XXVII SEMISH. Anais. Curitiba-Paraná, 2000.

[30] Silva, E.A.A. "Métricas para Hiperdocumentos Web: uma Análise Utilizando Técnicas de Aprendizado de Máquina" Dissertação de Mestrado. ICMC-USP, São Carlos -SP. Setembro de 2001. 77p.

[31] Silva, E.A.; Fortes, R.P.M. Web Structure Metrics: an Analysis Using Machine Learning Systems. ASSE'2001 (Second Argentine Symposium on Software Engineering) 30th Argentine Conference on Computer Science and Operational Research (JAIIO 2001). Proceedings. Buenos Aires, Argentina, setembro 2001. p.61-72 (Disponível em http://www.ing.unlpam.edu.ar/jaiio2001/) 